\title{
Acupuncture: Emerging evidence for its use as an analgesic (Review)
}

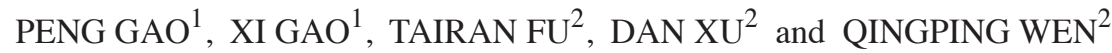 \\ ${ }^{1}$ Department of Anesthesiology, Dalian Medical University, Dalian, Liaoning 116044; ${ }^{2}$ Department of Anesthesiology, \\ First Affiliated Hospital of Dalian Medical University, Dalian, Liaoning 116011, P.R. China
}

Received June 27, 2014; Accepted February 18, 2015

DOI: 10.3892/etm.2015.2348

\begin{abstract}
Acupuncture is an ancient Chinese technique, developed over $>3,000$ years, in which 'acupoints' are stimulated with the aim of treating various diseases. A number of previous studies have indicated that acupuncture may play a role in inducing analgesia. Acupuncture-induced analgesia has been hypothesized to act on various parts of the central nervous system, including the spinal cord, brain stem, cerebral ganglia and cerebral cortex. The mechanisms underlying the effects of acupuncture have been purported to include neurohumors and neurotransmitters, such as opioids and $\gamma$-aminobutyric acid, signaling pathways and the immune response, which are all involved in the induction of analgesia.
\end{abstract}

\section{Contents}

1. Introduction

2. Acupuncture-induced analgesia may act on different levels of the nervous system

3. Proposed mechanisms of acupuncture-induced analgesia

4. Conclusion

\section{Introduction}

Acupuncture is an ancient Chinese technique that has been developed over a period of $>3,000$ years, in which 'acupoints' are stimulated to treat a variety of diseases. Acupuncture has numerous functions (1-3), including use as an auxiliary method of treatment for acute or chronic pain $(4,5)$. Certain studies have proposed that acupuncture-induced analgesia is a complex physiological adjustment process that involves the entire nervous system, including the central nervous system

Correspondence to: Professor Qingping Wen, Department of Anesthesiology, First Affiliated Hospital of Dalian Medical University, 222 Zhongshan Road, Dalian, Liaoning 116011, P.R. China

E-mail: dmuwqp@163.com

Key words: acupuncture, analgesia, nervous system
(CNS) and the peripheral nervous system (6-8). Mechanisms underlying acupuncture are intimately associated with neurohumoral and neurotransmitter pathways. A number of studies have hypothesized that acupuncture may play a role in inducing analgesia (8-11).

\section{Acupuncture-induced analgesia may act on different levels of the nervous system}

Acupuncture-induced analgesia is widely acknowledged to be associated with the functions of the entire nervous system, including the spinal cord, brain stem, limbic system and the cerebral cortex. Acupuncture-induced effects interact with and coordinate different levels of the CNS. The effect of acupuncture-induced analgesia is considered to be a complicated process (12-30).

Acupuncture and the spinal cord. The spinal cord is the first station of nociceptive information processing and interpretation, in addition to functioning as a pain reflex center. Peripheral nociception involves transmission into the spinal cord via the dorsal root, followed by sorting and analyzing by the spinal cord. The spinal cord is hypothesized to be the primary level of acupuncture analgesia, and the underlying mechanism may be associated with the release of certain neurotransmitters, such as 5-hydroxytryptamine (5-HT), somatostatin and substance P (SP) (12-14). Furthermore, opioid peptides may be involved in acupuncture analgesia in the spinal cord to varying degrees (15). Pharmacological inhibition of ascending nociceptive control at the spinal cord, nucleus accumbens or supraspinal may prevent acupuncture-induced analgesia (16).

Acupuncture and the brain stem. The brain stem is the relay station for sorting, discriminating and synthesizing information associated with pain. A proposed mechanism underlying acupuncture-induced analgesia in the brain stem involves the activation of the reticular formation of the brain stem descending pain-inhibitory system. The majority of this system is located in the periaqueductal gray and medulla oblongata nucleus raphe magnus, and is associated with a variety of neurotransmitters involved in analgesia, such as 5 -HT and opioid peptides $(17,18)$. A previous study demonstrated that damage to the locus coeruleus may improve the analgesic efficacy of acupuncture (19). 
Acupuncture and the cerebral ganglia. The cerebral ganglia is involved in analyzing and adjusting various types of information, and is modulated by a number of neurohumoral substances. These substances may be central for improving the efficacy of acupuncture-induced analgesia. Habenula nuclei serve key functions in monitoring pain, and activation of these structures may result in the inhibition of the raphe nuclei, which may in turn excite the locus coeruleus $(20,21)$. Norepinephrine and acetylcholine are generated from the locus coeruleus or habenula nuclei; they can improve the activities of these nuclei by interacting with each other and are also involved in acupuncture analgesia (18,22-24). The arcuate nucleus, paraventricular nucleus and preoptic area are located within the hypothalamus, and are associated with the modulation of antinociception to nociception (25-27). There are a number of possible mechanisms to explain this modulation, including the activation of the periaqueductal gray-nucleus raphe magnus pathway, excitation of the dorsal raphe nucleus and inhibition of the locus coeruleus (27). In addition, the preoptic area contains numerous neurotransmitters, among which opioid peptides and noradrenaline serve particularly crucial roles for analgesia (28).

Acupuncture and the cerebral cortex. The cerebral cortex is the center of higher nervous activity, including consciousness, intelligence, analysis and synthesis, reasoning and judgment. In the cerebral cortex, the effect of acupuncture-induced analgesia is not a simple excitatory or inhibition process, but a complex adjustment process $(25,29,30)$. The cumulative effect of repeated acupuncture stimulation was consistent with the characteristic habituation effects associated with functional magnetic resonance imaging examination (8).

\section{Proposed mechanisms of acupuncture-induced analgesia}

Acupuncture and opioids. Previous studies have suggested that acupuncture-induced analgesia is mediated by the release of endogenous opioids (31). Locally administered exogenous opioid receptor agonists bind to opioid receptors to produce analgesia. Acupuncture has been hypothesized to generate an increased release of endogenous opioids, and increase receptor affinity and/or number (32). Opioid peptides bind to their receptors on central neurons and subsequently induce antinociception (33).

The expression of all four subsets of opioid peptides, including endomorphin ( $\mu$-opioid agonist), dynorphin ( $\kappa$-opioid agonist),enkephalinand $\beta$-endorphin ( $\mu$-and $\delta$-opioidagonists), may be upregulated following acupuncture treatment (34); however, $\beta$-endorphin appears to be the most abundant opioid in acupuncture patients (35). Furthermore, the peripheral release of opioids may be involved in acupuncture-induced analgesia (36). There are multiple lines of evidence that suggest the possibility of acupuncture-induced peripheral opioid release. Firstly, acupuncture has been observed to modulate immunological activities (37), and immune cells are key components of the peripheral opioid system. Secondly, central opioid mechanisms present no evident mechanism through which needling an acupuncture point adjacent to a painful area may be able to reduce pain; however, central sensitization includes the malfunction of descending pain inhibitory pathways, which may result in dysfunctional endogenous analgesia (38). Thirdly, in clinical practice, acupuncture is generally more effective in relieving local pain compared with needling distal areas, a phenomenon that is comparable with the presence of a localized analgesic mechanism (39-41). Due to the lack of endogenous analgesia, increased temporal summation of secondary pain may contribute to the development or maintenance of central sensitization in patients with chronic pain (42). However, endogenous opioids released by acupuncture may induce analgesia and prevent the development of chronic pain (42).

Acupuncture and $\gamma$-aminobutyric acid (GABA). Certain studies have hypothesized that pain sensation may be suppressed by acupuncture, and the regulation of the glutamatergic and the GABAergic systems has been proposed as a possible mechanism (43. Inhibition of the excitatory glutamatergic system and stimulation of the inhibitory system contribute to antinociception. Stimulation of the GABA-A and GABA-B receptors has been demonstrated to result in pain suppression $(44,45)$.

Peripheral C-fiber stimulation activates inhibitory spinal mechanisms that are dependent on $\mu$-opioid and GABA receptors, which suppress ongoing ascending activity and induce analgesia via an opioidergic connection to the nucleus accumbens (46). GABA transporter 1 (GAT1) has been demonstrated to be involved in pain sensation in transgenic mice with knockout or overexpression of GAT1 (47). Activation of the $\delta$-opioid receptor may reduce the rate of GABA uptake and GAT1-mediated current. In a previous study, $\mu$-opioid receptor activation did not affect the number of transporters, the rate of GABA uptake or GAT1-mediated current (48). However, inhibition of GAT1-mediated current via the activation of the $\delta$-opioid receptor has been demonstrated in whole-cell patch-clamp experiments with rat brain slices of the periaqueductal gray. Therefore, the inhibition of GAT1 function may enhance the inhibitory effects of the GABAergic system and contribute to acupuncture-induced analgesia (49).

Acupuncture and signaling pathways. During nerve injury, phospho-P38 mitogen-activated protein kinase (p-P38 MAPK) levels have been observed to sequentially increase in the neurons, microglia and astrocytes of the spinal dorsal horn (49,50). In addition, nerve injury-induced upregulation of p-P38 MAPK may occur rapidly and persist for an extended period. Previous studies have demonstrated a significant increase in the levels of p-P38 MAPK during spinal dorsal horn-induced inflammation $(51,52)$. Furthermore, studies have indicated that p-P38 MAPK levels are increased in the spinal cord following application of complete Freund's adjuvant (CFA), and the administration of the P38 inhibitor prevents inflammation $(53,54)$. It has been indicated that the numbers of p-P38 MAPK-immunoreactive cells in the spinal dorsal horn increase rapidly and peak in a short time following CFA injection, and are maintained over an extended period (54).

Acupuncture may provoke extracellular signal-regulated kinase (ERK) activation in fibroblasts and keratinocytes of the dermal layer, and this analgesic effect has been demonstrated to be blocked when the ERK cascade is interrupted following administration of the MEK/MAPK inhibitor, U0126 (55). 
The ERK signaling pathway has been regarded as a marker for central sensitization (56). Cheng et al suggested that the protein levels of p-ERK1/2 were slightly increased and attenuated nociception in acupuncture-treated rats (57). A number of time-dependent effects have been observed as a result of MAPK-ERK1/2 pathway activation in the peripheral and CNS following acupuncture-induced analgesia (58).

Acupuncture and the immune system. Cytokines exert a central analgesic effect, the underlying mechanism of which may be associated with neuropeptide receptors (59). The immune system is able to participate in the occurrence of pain and signal transmission. A 'neuropeptide-cytokine network' has been hypothesized as a possible mechanism underlying acupuncture-induced analgesia and immune regulation (60). In this network of associations between neuropeptides and cytokines, neuropeptides may affect the differentiation and development of immune cells, which may in turn cause the synthesis and secretion of neuropeptides $(61,62)$. Thus, cytokines are not only key immunoregulatory factors, but also participate in pain signal conduction. In addition, cytokines are able to modulate the extent of neuropeptide secretion (63). Acupuncture has been hypothesized to stimulate receptors on complex structures, such as nerves, blood vessels and lymph vessels, in the acupuncture-point area $(64,65)$. Acupuncture has an effect on all levels of the 'nervous immune network'. It initiates neuropeptide production, cytokine processing and integration, and causes nerve impulses to pass through the peripheral and autonomic nerve system, thus generating a complete and precise regulation of the 'neuropeptide cytokine network.' Therefore, certain studies have suggested that acupuncture may exert an effect on pain and immune regulation (66-68).

5-HT serves a crucial function in specific physical and emotional responses. For example, low levels of 5-HT have been associated with anxiety, depression and stress (69). 5-HT is a neuroactive substance that participates in analgesic activity in the descending pain-inhibitory system of the brain stem, and it has been proposed that acupuncture affects 5-HT activity in the central neurons. A previous study suggested that the levels of 5-HT were correlated with acupuncture analgesia (70). 5-HT and its receptors in the CNS are known to be associated with the modulation of nociceptors (71). It was hypothesized that the lower pain scoring and increased friendliness observed in acupuncture-treated dogs may be due to increased acupuncture-induced secretion of 5-HT $(70,71)$.

Cholecystokinin octapeptide (CCK-8) and/or CCK receptors have been reported to be involved in acupuncture-induced analgesia. CCK- 8 has been demonstrated to possess notable anti-opioid activity at the spinal level. Thus, inhibition of the spinal cholecystokinin effect may be expected to potentiate opiate analgesia (72). Opioid activity in vivo is able to increase the expression of CCK-8, resulting in negative feedback regulation (73). Exogenous and endogenous opioid substances are able to promote the central release of CCK-8. A previous study demonstrated that systemic morphine produces a marked increase in CCK-8 immunoreactivity in the perfusate of the rat spinal cord, an effect that is reversed by naloxone (74). Opioid agonists of the $\kappa$ - and $\mu$-opioid receptors are known to increase CCK- 8 release, while the effects of $\alpha$-agonists are invalid (75). Furthermore, nociception may result in the development of serious pain due to the release of mediators, such as SP (76). Immunohistochemical studies have suggested that acupuncture depresses the pain response and increases SP-immunoreactivity, possibly due to the inhibition of SP release (77). Therefore, the inhibition of SP may reduce early pain perception.

\section{Conclusion}

Acupuncture is an ancient medical technology and comes from developed practises. Although preliminary, the neurobiological findings of previous studies have suggested a number of potential underlying mechanisms for an association between acupuncture and analgesia $(78,79)$. Therefore, the application of acupuncture for analgesia is a useful method in clinical practice.

\section{Acknowledgements}

The study was supported by a grant from the National Nature Science Fund of China (no. 81273923).

\section{References}

1. Yang L, Yue N, Zhu X, Han Q, Li B, Liu Q, Wu G and Yu J: Electroacupuncture promotes proliferation of amplifying neural progenitors and preserves quiescent neural progenitors from apoptosis to alleviate depressive-like and anxiety-like behaviours. Evid Based Complement Alternat Med 2014: 872568, 2014.

2. Xu T, Li W, Liang Y, Yang Z, Liu J, Wang Y and Su N: Neuroprotective effects of electro acupuncture on hypoxic-ischemic encephalopathy in newborn rats Ass. Pak J Pharm Sci 27 (6 Suppl): 1991-2000, 2014.

3. Yu J and Zhou J: Effect of auricular point sticking on lactation of puerperant. Zhongguo Zhen Jiu 32: 1087-1089, 2012 (In Chinese).

4. Bigeleisen PEand Goehner N: Novel approaches in pain management in cardiac surgery. Curr Opin Anaesthesiol 28: 89-94, 2015.

5. Hinman RS, McCrory P, Pirotta M, et al: Acupuncture for chronic knee pain: A randomized clinical trial. JAMA 312: 1313-1322, 2014.

6. Wu C, Qu S, Zhang J, Chen J, Zhang S, Li Z, Chen J, Ouyang H, Huang Y and Tang C: Correlation between the effects of acupuncture at Taichong (LR3) and functional brain areas: A resting-state functional magnetic resonance imaging study using true versus sham acupuncture. Evid Based Complement Alternat Med 2014: 729091, 2014.

7. Fang JQ, Fang JF, Liang Y and Du JY: Electroacupuncture mediates extracellular signal-regulated kinase $1 / 2$ pathways in the spinal cord of rats with inflammatory pain. BMC Complement Altern Med 14: 285, 2014.

8. Li C, Yang J Park K, Wu H, Hu S, Zhang W, Bu J, Xu C, Qiu B and Zhang X: Prolonged repeated acupuncture stimulation induces habituation effects in pain-related brain areas: An FMRI study. PLoS One 9: e97502, 2014.

9. Kim KH, Ryu JH, Park MR, et al: Acupuncture as analgesia for non-emergent acute non-specific neck pain, ankle sprain and primary headache in an emergency department setting: A protocol for a parallel group, randomised, controlled pilot trial. BMJ Open 4: e004994, 2014.

10. Moré AO, Cidral-Filho FJ, Mazzardo-Martins L, Martins DF, Nascimento FP, Li SM and Santos AR: Caffeine at moderate doses can inhibit acupuncture-induced analgesia in a mouse model of postoperative pain. J Caffeine Res 3: 143-148, 2013.

11. Grillo CM, Wada RS and da Luz Rosário de Sousa M: Acupuncture in the management of acute dental pain. J Acupunct Meridian Stud 7: 65-70, 2014.

12. Ruan H, Li X and Cai W: Effect of 5-HT and somatostatin on SP and chronic pain initiated electrical activity of neurons in spinal dorsal horn. Zhen Ci Yan Jiu 21: 27-31, 1996 (In Chinese). 
13. Qiao LN, Yang YS, Wang JY, Gao YH, Han YJ, Chen SP, Ji CF and Liu JL: Effects of electroacupuncture at "Futu" (LI 18), etc. on expression of spinal 5-HT 1 AR mRNA, 5-HT 2 AR mRNA and protein in rats with neck incision pain. Zhen Ci Yan Jiu 36: 391-396, 2011.

14. Schaible HG: Basic mechanisms of deep somatic pain. In: Wall and Melzack's Textbook of Pain. McMahon SB and Koltzenburg M (eds). Churchill-Livingston, London, pp621-633, 2006.

15. Zyloney CE, Jensen K, Polich G, Loiotile RE, Cheetham A, LaViolette PS, Tu P, Kaptchuk TJ, Gollub RL and Kong J: Imaging the functional connectivity of the Periaqueductal Gray during genuine and sham electroacupuncture treatment. Mol Pain 6: 80, 2010.

16. Tobaldini G, Aisengart B, Lima MMS, Tambeli $\mathrm{CH}$ and Fischer L: Ascending nociceptive control contributes to the antinociceptive effect of acupuncture in a rat model of acute pain. J Pain 15: 422-434, 2014.

17. Lee CH, Jung HS, Lee TY, Lee SR, Yuk SW, Lee KG and Lee BH: Studies of the central neural pathways to the stomach and Zusanli (ST36). Am J Chin Med 29: 211-220, 2001.

18. Zhang Y, Zhang RX, Zhang M, Shen XY, Li A, Xin J, Ren K, Berman BM, Tan M and Lao L: Electroacupuncture inhibition of hyperalgesia in an inflammatory pain rat model: Involvement of distinct spinal serotonin and norepinephrine receptor subtypes. Br J Anaesth 109: 245-252, 2012.

19. Kim KW, Kim HW, Li J and Kwon YB: Effect of bee venom acupuncture on methamphetamine-induced hyperactivity, hyperthermia and Fos expression in mice. Brain Res Bull 84: 61-68, 2011.

20. Shelton L, Pendse G, Maleki N, Moulton EA, Lebel A, Becerra L and Borsook D: Mapping pain activation and connectivity of the human habenula. J Neurophysiol 107: 2633-2648, 2012.

21. Liu GJ and Wang S: Effects of nucleus raphe magnus and locus coeruleus in descending modulation of the habenula on pain threshold and acupuncture analgesia. Zhongguo Yao Li Xue Bao 9: 18-22, 1988 (In Chinese).

22. Wang $\mathrm{H}$, Jiang $\mathrm{J}$ and Can $\mathrm{X}$ : Changes of norepinephrine release in rat's nucleus reticularis paragigantocellularis lateralis in acupuncture analgesia. Zhen Ci Yan Jiu 19: 20-25, 1994 (In Chinese)

23. Wang JY, Meng FY, Chen SP, Gao YH and Liu JL: Analysis on interrelation between electroacupuncture-induced cumulative analgesic effect and hypothalamic cholinergic activities in chronic neuropathic pain rats. Chin J Integr Med 18: 699-707, 2012.

24. Guan X, Wang C, Yu B, Liang X, Zhang Y, Zeng X, Liu X, Shi J and Ai M: Research on the relationship between central acetylcholine and acupuncture analgesia. Zhen Ci Yan Jiu 16: 129-137, 1991 (In Chinese).

25. Wang JY, Chen SP, Li YH, Meng FY, Gao YH and Liu JL: Observation on the accumulative analgesic effect of electroacupuncture and the expression of protein kinase A in hypothalamus and hippocampus in chronic pain or/and ovariectomized rats. Zhen Ci Yan Jiu 33: 80-87, 2008 (In Chinese).

26. Xu Q, Liu T, Chen S, Gao Y, Wang J, Qiao L and Liu J: Correlation between the cumulative analgesic effect of electroacupuncture intervention and synaptic plasticity of hypothalamic paraventricular nucleus neurons in rats with sciatica. Neural Regen Res 8: 218-225, 2013.

27. Zhu JM, Li KY and Cao XD: Effects of destruction of preoptic catecholaminergic nerve terminals on acupuncture analgesia. Sheng Li Xue Bao 42: 135-140, 1990 (In Chinese).

28. Wang SJ, Zhu B, Ren XX, Ben H, Li YQ and Li YH: Effect of acupuncture of different acupoints on electrical activities of hypothalamic sexual arousal stimulation-related neurons at different stages of oestrous cycle in rats. Zhen Ci Yan Jiu 32: 313-318, 2007 (In Chinese)

29. Newberg AB, Lariccia PJ, Lee BY, Farrar JT, Lee L and Alavi A: Cerebral blood flow effects of pain and acupuncture A preliminary single-photon emission computed tomography imaging study. J Neuroimaging 15: 43-49, 2005.

30. Yi M, Zhang H, Lao L, Xing GG and Wan Y: Anterior cingulate cortex is crucial for contra- but not ipsi-lateral electro-acupuncture in the formalin-induced inflammatory pain model of rats. Mol Pain 7: 61, 2011.

31. Li J, Zhang JH, Yi T, Tang WJ, Wang SW and Dong JC: Acupuncture treatment of chronic low back pain reverses an abnormal brain default mode network in correlation with clinical pain relief. Acupunct Med 32: 102-108, 2014
32. Huang C, Wang Y, Chang JK and Han JS: Endomorphin and mu-opioid receptors in mouse brain mediate the analgesic effect induced by $2 \mathrm{~Hz}$ but not $100 \mathrm{~Hz}$ electroacupuncture stimulation. Neurosci Lett 294: 159-162, 2000.

33. Harris RE, Zubieta JK, Scott DJ, Napadow V, Gracely RH and Clauw DJ: Traditional Chinese acupuncture and placebo (sham) acupuncture are differentiated by their effects on mu-opioid receptors (MORs). Neuroimage 47: 1077-1085, 2009.

34. Pasternak GW: Pharmacological mechanisms of opioid analgesics. Clin Neuropharmacol 16: 1-18, 1993.

35. Han JS: Acupuncture and endorphins. Neurosci Lett 361: 258-261, 2004.

36. Zhang GG, Yu C, Lee W, Lao L, Ren K and Berman BM: Involvement of peripheral opioid mechanisms in electroacupuncture analgesia. Explore (NY) 1: 365-371, 2005.

37. Cabot PJ, Carter L, Schäfer $M$ and Stein C Methionine-enkephalin and Dynorphin A-release from immune cells and control of inflammatory pain. Pain 93: 207-212, 2001

38. Cheng XD, Wu GC, He QZ and Cao XD: Effect of continued electroacupuncture on induction of interleukin-2 production of spleen lymphocytes from the injured rats. Acupunct Electrother Res 22: 1-8, 1997.

39. Miao EY: Clinical critical qualitative evaluation of the selected randomized controlled trials in current acupuncture researches for low back pain. Zhong Xi Yi Jie He Xue Bao 8: 1133-1146, 2010 (In Chinese)

40. Weiss J, Quante S, Xue F, Muche R and Reuss-Borst M: Effectiveness and acceptance of acupuncture in patients with chronic low back pain: Results of a prospective, randomized, controlled trial. J Altern Complement Med 19: 935-941, 2013.

41. Goldman N, Chen M, Fujita T, et al: Adenosine A1 receptors mediate local anti-nociceptive effects of acupuncture. Nat Neurosci 13: 883-888, 2010.

42. Meeus M, Nijs J, Van de Wauwer N, Toeback L and Truijen S: Diffuse noxious inhibitory control is delayed in chronic fatigue syndrome: An experimental study. Pain 139: 439-448, 2008.

43. Cao W, Deng Y, Dong X, Wang Y and Lu Z: Effects of electroacupuncture at different frequencies on the nociceptive response and central contents of GABA and glutamic acid in arthritic rats. Zhen Ci Yan Jiu 18: 48-52, 1993 (In Chinese).

44. Li X, Zhou K, Zhang E, Qi Z, Sun W, Xu L, Xu J, Cai Y and Wang R: Therapeutic effect of electroacupuncture, massage, and blocking therapy on external humeral epicondylitis. J Tradit Chin Med 34: 261-266, 2014

45. Malan TP Jr, Mata HP and Porreca F: Spinal GABA(A) and GABA(B) receptor pharmacology in a rat model of neuropathic pain. Anesthesiology 96: 1161-1167, 2002.

46. Knabl J, Witschi R, Hösl K, et al: Reversal of pathological pain through specific spinal GABAA receptor subtypes. Nature 451: 330-334, 2008

47. Tambeli CH, Quang P, Levine JD and Gear RW: Contribution of spinal inhibitory receptors in heterosegmental antinociception induced by noxious stimulation. Eur J Neurosci 18: 2999-3006, 2003.

48. Hu JH, Yang N, Ma YH, Zhou XG, Jiang J, Duan SH, Mei ZT, Fei $J$ and Guo LH: Hyperalgesic effects of $\gamma$-aminobutyric acid transporter I in mice. J Neurosci Res 73: 565-572, 2003.

49. Moon JY, Roh DH, Yoon SY, et al: o1 receptors activate astrocytes via p38 MAPK phosphorylation leading to the development of mechanical allodynia in a mouse model of neuropathic pain. Br J Pharmacol 171: 5881-5897, 2014.

50. Gong QJ, Li YY, Xin WJ, Zang Y, Ren WJ, Wei XH, Li YY, Zhang T and Liu XG: ATP induces long-term potentiation of C-fiber-evoked field potentials in spinal dorsal horn: The roles of P2X4 receptors and p38 MAPK in microglia. Glia 57: 583-591, 2009.

51. Pu L, Xu N, Xia P, Gu Q, Ren S, Fucke T, Pei G and Schwarz W: Inhibition of activity of GABA transporter GAT 1 by $\delta$-opioid receptor. Evid Based Complement Alternat Med 2012: 818451, 2012.

52. Cui XY, Dai Y, Wang SL, Yamanaka H, Kobayashi K, Obata K, Chen J and Noguchi K: Differential activation of p38 and extracellular signal-regulated kinase in spinal cord in a model of bee venom-induced inflammation and hyperalgesia. Mol Pain 4: 17, 2008.

53. Zaringhalam J, Tekieh E, Manaheji $\mathrm{H}$ and Akhtari Z: Cellular events during arthritis-induced hyperalgesia are mediated by interleukin-6 and p38 MAPK and their effects on the expression of spinal mu-opioid receptors. Rheumatol Int 33: 2291-2299, 2013. 
54. Fang JQ, Du JY, Liang Y and Fang JF: Intervention of electroacupuncture on spinal p38 MAPK/ATF-2/VR-1 pathway in treating inflammatory pain induced by CFA in rats. Mol Pain 9: 13, 2013.

55. Boyle DL, Jones TL, Hammaker D, Svensson CI, Rosengren S, Albani S, Sorkin L and Firestein GS: Regulation of peripheral inflammation by spinal p38 MAP kinase in rats. PLoS Med 3: e338, 2006

56. Park JY, Park JJ, Jeon S, Doo AR, Kim SN, Lee H, Chae Y, Maixner W, Lee H and Park HJ: From peripheral to central: The role of ERK signaling pathway in acupuncture analgesia. J Pain 15: 535-549, 2014.

57. Cheng SJ, Chen CC, Yang HW, Chang YT, Bai SW, Chen CC Yen CT and Min MY: Role of extracellular signal-regulated kinase in synaptic transmission and plasticity of a nociceptive input on capsular central amygdaloid neurons in normal and acid-induced muscle pain mice. J Neurosci 31: 2258-2270, 2011.

58. Lu J, Liang J, Wang JR, Hu L, Tu Y and Guo JY: Acupuncture activates ERK-CREB pathway in rats exposed to chronic unpredictable mild stress. Evid Based Complement Alternat Med 2013: 469765, 2013.

59. Wang SJ, Yang HY and Xu GS: Acupuncture alleviates colorectal hypersensitivity and correlates with the regulatory mechanism of TrpV1 and p-ERK. Evid Based Complement Alternat Med 2012: 483123, 2012.

60. Shi X, Wang L, Clark JD and Kingery WS: Keratinocytes express cytokines and nerve growth factor in response to neuropeptide activation of the ERK1/2 and JNK MAPK transcription pathways. Regul Pept 186: 92-103, 2013.

61. Sekido R, Ishimaru K and Sakita M: Corticotropin-releasing factor and interleukin-1beta are involved in the electroacupuncture-induced analgesic effect on inflammatory pain elicited by carrageenan. Am J Chin Med 32: 269-279, 2004.

62. Gonzalez-Rey E, Chorny A, Fernandez-Martin A, Ganea D and Delgado M: Vasoactive intestinal peptide generates human tolerogenic dendritic cells that induce CD4 and CD8 regulatory T cells. Blood 107: 3632-3638, 2006.

63. Atanassova N, McKinnell C, Fisher J and Sharpe RM: Neonatal treatment of rats with diethylstilboestrol (DES) induces stromal-epithelial abnormalities of the vas deferens and cauda epididymis in adulthood following delayed basal cell development. Reproduction 129: 589-601, 2005.

64. Huang T, Yang L, Jia S, Mu X, Wu M, Ye H, Liu W and Cheng X: Capillary blood flow in patients with dysmenorrhea treated with acupuncture. J Tradit Chin Med 33: 757-760, 2013

65. Li ZZ, Chen JZ, Guo Y, Guo YM and Liu YY: Influence of acupuncture or thermal acupuncture stimulation at 'Zusanli' (ST 36) on thoracic duct lymph volume and the relevant chemical substances in normal rats. Zhen Ci Yan Jiu 37: 453-457, 2012 (In Chinese)

66. Fu X, Zhu ZH, Wang YQ and Wu GC: Regulation of proinflammatory cytokines gene expression by nociceptin/orphanin FQ in the spinal cord and the cultured astrocytes. Neuroscience 144 $275-285,2007$
67. Gao YH, Wang JY, Qiao LN, Chen SP, Tan LH, Xu QL and Liu JL: NK cells mediate the cumulative analgesic effect of electroacupuncture in a rat model of neuropathic pain. BMC Complement Altern Med 14: 316, 2014.

68. Peng G: Acupuncture and innate immunity. Zhen Ci Yan Jiu 33 49-52, 2008 (In Chinese)

69. Jeong HJ, Hong SH, Nam YC, Yang HS, Lyu YS, Baek SH, Lee HJ and Kim HM: The effect of acupuncture on proinflammatory cytokine production in patients with chronic headache: A preliminary report. Am J Chin Med 31: 945-954, 2003.

70. Yu JN, Guo CQ, Hu B, Liu NG, et al: Effects of acupuncture knife on inflammatory factors and pain in third lumbar vertebrae transverse process syndrome model rats. Evid Based Complement Alternat Med 2014: 892406, 2014.

71. Erthal V, da Silva MD, Cidral-Filho FJ, Santos AR and Nohama P: ST36 laser acupuncture reduces pain-related behavior in rats: Involvement of the opioidergic and serotonergic systems. Lasers Med Sci 28: 1345-1351, 2013

72. Kayser V, Elfassi IE, Aubel B, Melfort M, Julius D, Gingrich JA, Hamon $M$ and Bourgoin S: Mechanical, thermal and formalin-induced nociception is differentially altered in 5-HT1A-/-, 5-HT1B-/-, 5-HT2A-/-, 5-HT3A-/- and 5-HTT-/knock-out male mice. Pain 130: 235-248, 2007.

73. Huang C, Hu ZP, Jiang SZ, Li HT, Han JS and Wan Y: CCK(B) receptor antagonist L365,260 potentiates the efficacy to and reverses chronic tolerance to electroacupuncture-induced analgesia in mice. Brain Res Bull 71: 447-451, 2007.

74. Li M, Tjen-A-Looi SC, Guo ZL and Longhurst JC: Electroacupuncture modulation of reflex hypertension in rats: Role of cholecystokinin octapeptide. Am J Physiol Regul Integr Comp Physiol 305: R404-R413, 2013.

75. Yamada Y, Ohinata K, Lipkowski AW and Yoshikawa M: Rapakinin, Arg-Ile-Tyr, derived from rapeseed napin, shows anti-opioid activity via the prostaglandin IP receptor followed by the cholecystokinin CCK(2) receptor in mice. Peptides 32: 281-285, 2011

76. Romanelli L, Morrone LA, Amico MC, Palmery M, Tucci P and Valeri P: Inhibitory control of the acute mu-withdrawal response by indirectly activated adenosine A1 and kappa-opioid systems in the Guinea-pig ileum; reversal by cholecystokinin. Neurotoxicology 26: 829-839, 2005.

77. Du J and He L: Alterations of spinal dorsal horn substance P following electroacupuncture analgesia - A study of the formalin test with immunohistochemistry and densitometry. Acupunct Electrother Res 17: 1-6, 1992

78. Huang B, Zhou ZL, Wang LL, Zuo C, Lu Y and Chen Y: Electrical response grading versus House-Brackmann scale for evaluation of facial nerve injury after Bell's palsy: A comparative study. J Integr Med 12: 367-371, 2014.

79. Shokrani O, Saghaei M, Ashrafi F and Sadeghi A: Electrical stimulation of acupuncture points for analgesia during bone marrow aspiration and biopsy: A randomized double-blind placebo-controlled trial. Adv Biomed Res 3: 125, 2014. 